\title{
The Existence of Meromorphic Solutions of Some Types of Systems of Complex Functional Equations
}

\author{
Hong-Yan Xu, ${ }^{1}$ Jin-Lian Wang, ${ }^{2}$ and Hua Wang' \\ ${ }^{1}$ Department of Informatics and Engineering, Jingdezhen Ceramic Institute, Jingdezhen, Jiangxi 333403, China \\ ${ }^{2}$ Jiangxi Normal University, Nanchang, Jiangxi 330027, China \\ Correspondence should be addressed to Hong-Yan Xu; xhyhhh@126.com
}

Received 4 August 2015; Accepted 13 September 2015

Academic Editor: Chris Goodrich

Copyright (c) 2015 Hong-Yan Xu et al. This is an open access article distributed under the Creative Commons Attribution License, which permits unrestricted use, distribution, and reproduction in any medium, provided the original work is properly cited.

\begin{abstract}
We investigate the existence of transcendental meromorphic solutions of some types of systems of complex functional equations and obtain some results about the existence of meromorphic solutions of such systems. Our results are improvement of the previous theorems given by $\mathrm{Gao}, \mathrm{Xu}$, and Zheng, and our examples show that our results are sharp to some extent.
\end{abstract}

\section{Introduction and Main Results}

Recently, with the establishment of the differences analogues of Nevanlinna's theory (see [1-3]), people obtained many interesting theorems about the growth and existence of solutions of difference equations, $q$-difference equations, and so on (see [4-10]). To state some results, we should introduce some basic definition and standard notations. We firstly assume that readers are familiar with the fundamental results and the standard notations of the Nevanlinna value distribution theory of meromorphic functions such as $m(r, f), N(r, f), T(r, f), \ldots$ (see Hayman [11], Yang [12], and Yi and Yang [13]). In addition, we also use $\rho(f), \lambda(f)$, and $\lambda(1 / f)$ to denote the order, the exponent of convergence of zeros, and the exponent of convergence of poles of $f(z)$, respectively, and $S(r, f)$ to denote any quantity satisfying $S(r, f)=o(T(r, f))$ for all $r$ outside a possible exceptional set $E$ of finite logarithmic measure $\lim _{r \rightarrow \infty} \int_{[1, r) \cap E}(d t / t)<\infty$, and a meromorphic function $a(z)$ is called small function with respect to $f$, if $T(r, a)=S(r, f)=o(T(r, f))$.

In 2001, Heittokangas et al. considered the existence of solutions of some difference equations and obtained the following results [14].
Theorem 1 (see [14, Propositions 8 and 9]). Let $c_{1}, \ldots, c_{n} \in$ $\mathbb{C} \backslash\{0\}$. If the following equations

$$
\begin{aligned}
& \sum_{j=1}^{n} f\left(z+c_{j}\right)=R(z, f(z)), \\
& \prod_{j=1}^{n} f\left(z+c_{j}\right)=R(z, f(z)),
\end{aligned}
$$

where

$$
\begin{aligned}
R(z, f(z)):=\frac{P(z, f(z))}{Q(z, f(z))} \\
=\frac{a_{0}(z)+a_{1}(z) f(z)+\cdots+a_{s}(z) f(z)^{s}}{b_{0}(z)+b_{1}(z) f(z)+\cdots+b_{t}(z) f(z)^{t}},
\end{aligned}
$$

with small coefficients $a_{i}(z), b_{j}(z)$ with respect to $f$, admit transcendental meromorphic solutions of finite order, then $\max \{s, t\} \leq n$.

Theorem 2 (see [14]). Let $c_{1}, \ldots, c_{n} \in \mathbb{C} \backslash\{0\}$. If (2) with small coefficients $a_{i}(z), b_{i}(z)$ of growth $S(r, f)$ such that $a_{s} b_{t} \neq \equiv 0$. If

$$
\max \left\{\lambda(f), \lambda\left(\frac{1}{f}\right)\right\}<\rho(f)
$$


then (2) is of the form

$$
\prod_{j=1}^{n} f\left(z+c_{j}\right)=c(z) f(z)^{k}
$$

where $c(z)$ is meromorphic, $T(r, c)=S(r, f)$, and $k \in \mathbb{Z}$.

In 2002, Gundersen et al. considered the solutions of $q$ difference equation and obtained the following result.

Theorem 3 (see [15]). Suppose that $f$ is a transcendental meromorphic solution of a q-difference equation of the form

$$
f(q z)=\frac{a_{0}(z)+a_{1}(z) f(z)+\cdots+a_{s}(z) f(z)^{s}}{b_{0}(z)+b_{1}(z) f(z)+\cdots+b_{t}(z) f(z)^{t}},
$$

where $q \in C,|q|>1, b_{t}(z) \equiv 1$, and meromorphic coefficients $a_{i}(z), b_{j}(z)$ are of growth $S(r, f)$. If

$$
\bar{N}(r, f)+\bar{N}\left(r, \frac{1}{f}\right)=S(r, f),
$$

then (6) is either of the form

$$
\begin{gathered}
f(q z)=a_{s}(z) f(z)^{s} \\
\text { or } f(q z)=a_{0}(z) f(z)^{-t} .
\end{gathered}
$$

In 2012, Zheng and Chen [16] further considered a $q$ difference equation more general than (2) under a condition similar to Theorem 2 on meromorphic solutions and obtained the following result.

Theorem 4 (see [16, Theorem 1]). Suppose that $f$ is a transcendental meromorphic solution of a q-difference equation of the form

$$
\begin{aligned}
\prod_{j=1}^{n} f\left(q_{j} z\right) & =R(z, f(z)) \\
& =\frac{a_{0}(z)+a_{1}(z) f(z)+\cdots+a_{s}(z) f(z)^{s}}{b_{0}(z)+b_{1}(z) f(z)+\cdots+b_{t}(z) f(z)^{t}},
\end{aligned}
$$

where $_{j} \in C \backslash\{0,1\}, j=1, \ldots, n$, and $R(z, f)$ is an irreducible rational function in $f$ with meromorphic coefficients $a_{i}(z)(i=$ $0, \ldots, s)$ and $b_{j}(z)(j=0, \ldots, t)$ of growth $S(r, f)$ such that $b_{t}(z) \equiv 1, a_{s}(z) \not \equiv 0$. If

$$
\max \left\{\lambda(f), \lambda\left(\frac{1}{f}\right)\right\}<\rho(f),
$$

then (9) is reduced to the form

$$
\begin{aligned}
\prod_{j=1}^{n} f\left(q_{j} z\right) & =a_{s}(z) f(z)^{s} \\
\operatorname{or} \prod_{j=1}^{n} f\left(q_{j} z\right) & =a_{0}(z) f(z)^{-t} .
\end{aligned}
$$

In 2012, Gao [17] studied the similar problem when (9) is replaced by the following system of functional equations,

$$
\begin{aligned}
& f_{1}(p(z))=R_{1}\left(z, f_{2}(z)\right)=\frac{\sum_{i=0}^{s_{2}} a_{i}(z) f_{2}(z)^{i}}{\sum_{j=0}^{t_{2}} b_{j}(z) f_{2}(z)^{j}}, \\
& f_{2}(p(z))=R_{2}\left(z, f_{1}(z)\right)=\frac{\sum_{i=0}^{s_{1}} c_{i}(z) f_{1}(z)^{i}}{\sum_{j=0}^{t_{1}} d_{j}(z) f_{1}(z)^{j}},
\end{aligned}
$$

where $p(z)$ is an entire function, $R_{1}\left(z, f_{2}(z)\right), R_{2}\left(z, f_{1}(z)\right)$ are irreducible rational functions, and the coefficients are small functions, and obtained the following theorems.

Theorem 5 (see [17, Theorem 1]). Let $\left(f_{1}, f_{2}\right)$ be a pair of nonconstant meromorphic solutions of system (12). Then, $p(z)$ is a polynomial.

Theorem 6 (see [17, Theorem 2]). Let the polynomial $p(z)=$ $q z^{k}+\cdots$ be of degree $k \geq 2,\left(f_{1}, f_{2}\right)$ a pair of transcendental meromorphic solutions of system (12), and $a_{s_{2}} b_{t_{2}} \not \equiv 0, c_{s_{1}} d_{t_{1}} \not \equiv$ $0, a_{i}, b_{j}, c_{i}, d_{j}$ small functions. If

$$
\begin{array}{r}
\bar{N}\left(r, f_{t}(p(z))\right)+\bar{N}\left(r, \frac{1}{f_{t}(p(z))}\right)=S(r, f) \\
t=1,2,
\end{array}
$$

then system (12) is of the form

$$
\begin{aligned}
& f_{1}(p(z))=a(z) f_{2}(z)^{s}, \\
& f_{2}(p(z))=b(z) f_{1}(z)^{t},
\end{aligned}
$$

where $a(z), b(z)$ are meromorphic, $T(r, a(z))=$ $S\left(r, f_{i}\right), \quad T(r, b(z))=S\left(r, f_{i}\right)$, and $s, t \in \mathbb{Z}, s \neq 0, t \neq 0$.

Remark 7. We can see that the numerical values of $s, t$ are uncertain in the conclusion of Theorem 6 .

After his works, there were some papers focusing on the growth and existence of meromorphic solutions of some types of systems of complex functional equations (see [1821]).

In this paper, we will further investigate some properties of solutions of the system of complex functional equations. The main purpose of this paper is to find out the relationship between " $s, t$ " and " $s_{i}, t_{j}$ " and further to confirm the numerical values of $s, t$ in the conclusion of Theorem 6 . Some results are obtained in this paper, and the first main result is about meromorphic solutions with few zeros and poles of a type of system of complex functional equations. 
Theorem 8. Let $q_{j_{t}} \in \mathbb{C} \backslash\{0,1\}, j_{t}=1, \ldots, n_{t}, t=1,2$, and let $\left(f_{1}, f_{2}\right)$ be a pair of nonrational meromorphic solutions of the system

$$
\begin{aligned}
& \prod_{j_{1}=1}^{n_{1}} f_{1}\left(q_{j_{1}} z\right) \\
& \quad=\frac{a_{0}(z)+a_{1}(z) f_{2}(z)+\cdots+a_{s_{2}}(z) f_{2}(z)^{s_{2}}}{b_{0}(z)+b_{1}(z) f_{2}(z)+\cdots+b_{t_{2}}(z) f_{2}(z)^{t_{2}}} \\
& \prod_{j_{2}=1}^{n_{2}} f_{2}\left(q_{j_{2}} z\right) \\
& \quad=\frac{e_{0}(z)+e_{1}(z) f_{1}(z)+\cdots+e_{s_{1}}(z) f_{1}(z)^{s_{1}}}{d_{0}(z)+d_{1}(z) f_{1}(z)+\cdots+d_{t_{1}}(z) f_{1}(z)^{t_{1}}}
\end{aligned}
$$

with the meromorphic coefficients $a_{i}(z), b_{i}(z), e_{i}(z), d_{i}(z)$ of growth $S\left(r, f_{t}\right), t=1,2$, and $a_{s_{2}}(z) e_{s_{1}}(z) \not \equiv 0, d_{t_{1}}(z) \equiv$ $b_{t_{2}}(z) \equiv 1$. If

$$
\max \left\{\lambda\left(f_{t}\right), \lambda\left(\frac{1}{f_{t}}\right)\right\}<\rho\left(f_{t}\right), \quad t=1,2,
$$

then system (15) is one of the four following forms:

$$
\begin{aligned}
& \prod_{j_{1}=1}^{n_{1}} f_{1}\left(q_{j_{1}} z\right)=a_{s_{2}}(z) f_{2}(z)^{s_{2}}, \\
& \prod_{j_{2}=1}^{n_{2}} f_{2}\left(q_{j_{2}} z\right)=e_{s_{1}}(z) f_{1}(z)^{s_{1}}, \\
& \prod_{j_{1}=1}^{n_{1}} f_{1}\left(q_{j_{1}} z\right)=a_{s_{2}}(z) f_{2}(z)^{s_{2}}, \\
& \prod_{j_{2}=1}^{n_{2}} f_{2}\left(q_{j_{2}} z\right)=e_{0}(z) f_{1}(z)^{-t_{1}}, \\
& \prod_{j_{1}=1}^{n_{1}} f_{1}\left(q_{j_{1}} z\right)=a_{0}(z) f_{2}(z)^{-t_{2}}, \\
& \prod_{j_{2}=1}^{n_{2}} f_{2}\left(q_{j_{2}} z\right)=e_{s_{1}}(z) f_{1}(z)^{s_{1}}, \\
& \prod_{j_{1}=1}^{n_{1}} f_{1}\left(q_{j_{1}} z\right)=a_{0}(z) f_{2}(z)^{-t_{2}}, \\
& \prod_{2}^{n_{2}} f_{2}\left(q_{j_{2}} z\right)=e_{0}(z) f_{1}(z)^{-t_{1}} .
\end{aligned}
$$

Corollary 9. Let $\left(f_{1}, f_{2}\right)$ be a pair of transcendental solutions of (15) with finite order $\rho\left(f_{1}\right)=\rho_{1}, \rho\left(f_{2}\right)=\rho_{2}, \rho\left(f_{1}\right), \rho\left(f_{2}\right)$ are positive integers and all the other assumptions of Theorem 8 hold.

(i) If $0<\rho_{1}<\rho_{2}<\infty$ and $\sum_{j_{2}=1}^{n_{2}} q_{j_{2}}^{\rho_{2}} \neq 0$, then system (15) is reduced to (18b) only. (ii) If $0<\rho_{2}<\rho_{1}<\infty$ and $\sum_{j_{1}=1}^{n_{1}} q_{j_{1}}^{\rho_{1}} \neq 0$, then system (15) is reduced to (18b) only.

Corollary 10. Let $\left(f_{1}, f_{2}\right)$ be a pair of transcendental solutions of (15) with finite order $\rho\left(f_{1}\right)=\rho_{1}, \rho\left(f_{2}\right)=\rho_{2}, \rho\left(f_{1}\right), \rho\left(f_{2}\right)$ are positive integers and all the other assumptions of Theorem 8 hold. If system (15) is reduced to (18b) only, and

$$
\left(\sum_{j_{2}=1}^{n_{2}} q_{j_{2}}^{\rho_{2}}+t_{2}\right)\left(\sum_{j_{1}=1}^{n_{1}} q_{j_{1}}^{\rho_{1}}+t_{1}\right) \neq 0
$$

then $\rho_{1}=\rho_{2}$.

Furthermore, we obtain the following result when system (15) is replaced by the other system.

Theorem 11. Let $q_{j_{t}}, c_{j_{t}} \in \mathbb{C} \backslash\{0,1\}, j_{t}=1, \ldots, n_{t}, t=1,2$, and let $\left(f_{1}, f_{2}\right)$ be a pair of nonrational meromorphic solutions of the system

$$
\begin{aligned}
& \prod_{j_{1}=1}^{n_{1}} f_{1}\left(q_{j_{1}} z+c_{j_{1}}\right) \\
& \quad=\frac{a_{0}(z)+a_{1}(z) f_{2} \circ p+\cdots+a_{s_{2}}(z)\left(f_{2} \circ p\right)^{s_{2}}}{b_{0}(z)+b_{1}(z) f_{2} \circ p+\cdots+b_{t_{2}}(z)\left(f_{2} \circ p\right)^{t_{2}}}, \\
& \prod_{j_{2}=1}^{n_{2}} f_{2}\left(q_{j_{2}} z+c_{j_{2}}\right) \\
& \quad=\frac{e_{0}(z)+e_{1}(z) f_{1} \circ p+\cdots+e_{s_{1}}(z)\left(f_{1} \circ p\right)^{s_{1}}}{d_{0}(z)+d_{1}(z) f_{1} \circ p+\cdots+d_{t_{1}}(z)\left(f_{1} \circ p\right)^{t_{1}}},
\end{aligned}
$$

with the meromorphic coefficients $a_{i}(z), b_{i}(z), e_{i}(z), d_{i}(z)$ of growth $S\left(r, f_{t}\right), t=1,2$, and $a_{s_{2}}(z) e_{s_{1}}(z) \quad \equiv \quad 0, d_{t_{1}}(z) \equiv$ $b_{t_{2}}(z) \equiv 1$. If $f_{1}, f_{2}$ satisfy (16) and $f \circ p=f(p(z))$, where $p(z)=q z^{k}+\cdots$ is a polynomial in $z$ of degree $k \geq 2$, then $f_{1}, f_{2}$ satisfy the following forms:

$$
\begin{aligned}
& \prod_{j_{1}=1}^{n_{1}} f_{1}\left(q_{j_{1}} z+c_{j_{1}}\right)=a_{s_{2}}(z)\left(f_{2} \circ p\right)^{s_{2}} \\
& \text { or } \prod_{j_{1}=1}^{n_{1}} f_{1}\left(q_{j_{1}} z+c_{j_{1}}\right)=a_{0}(z)\left(f_{2} \circ p\right)^{-t_{2}} \text {, } \\
& \prod_{j_{2}=1}^{n_{2}} f_{2}\left(q_{j_{2}} z+c_{j_{2}}\right)=e_{s_{1}}(z)\left(f_{1} \circ p\right)^{s_{1}} \\
& \text { or } \prod_{j_{2}=1}^{n_{2}} f_{2}\left(q_{j_{2}} z+c_{j_{2}}\right)=e_{0}(z)\left(f_{1} \circ p\right)^{-t_{1}} \text {. }
\end{aligned}
$$

The researches on the properties of solutions of complex differential equations in the whole complex plane, disc, and angular domain are always interesting in the past several decades (see [22-26]). In 2014, Liu and Dong [27] considered the existence of solutions of some differential-difference equation, where the differential-difference equation is an 
equation including derivatives, shifts, or differences of $f(z)$, and obtained the following result.

Theorem 12 (see [27, Theorem 2.3]). Let $k$ be a positive integer. If the equation

$$
\begin{aligned}
\sum_{k=1}^{n} f^{(k)}(z)=R(z, f(z+c)) \\
=\frac{a_{0}(z)+a_{1}(z) f(z+c)+\cdots+a_{p}(z) f(z+c)^{p}}{b_{0}(z)+b_{1}(z) f(z+c)+\cdots+b_{q}(z) f(z+c)^{q}}
\end{aligned}
$$

admits a transcendental meromorphic solution of finite order, then $\max \{p, q\} \leq n+1$. If (22) admits a transcendental entire solution of finite order, then $\max \{p, q\} \leq 1$.

Then, we will further consider the system of differentialdifference equations with the analogue form of (22) and obtain the following theorem.

Theorem 13. Let $k_{1}, k_{2}, n_{1}, n_{2}$ be integers. If system

$$
\begin{aligned}
& \sum_{k_{1}=1}^{n_{1}} f_{1}^{\left(k_{1}\right)}(z) \\
& =\frac{a_{0}(z)+a_{1}(z) f_{2}(z+c)+\cdots+a_{s_{2}}(z) f_{2}(z+c)^{s_{2}}}{b_{0}(z)+b_{1}(z) f_{2}(z+c)+\cdots+b_{t_{2}}(z) f_{2}(z+c)^{t_{2}}}, \\
& \sum_{k_{2}=1}^{n_{2}} f_{2}^{\left(k_{2}\right)}(z) \\
& =\frac{e_{0}(z)+e_{1}(z) f_{1}(z+c)+\cdots+e_{s_{1}}(z) f_{1}(z+c)^{s_{1}}}{d_{0}(z)+d_{1}(z) f_{1}(z+c)+\cdots+d_{t_{1}}(z) f_{1}(z+c)^{t_{1}}},
\end{aligned}
$$

with the meromorphic coefficients $a_{i}(z), b_{i}(z), e_{i}(z), d_{i}(z)$ of growth $S\left(r, f_{t}\right), t=1,2$, and $a_{s_{2}}(z) e_{s_{1}}(z) \neq \equiv$, admits a pair of transcendental meromorphic solutions of finite order, set $\sigma_{1}=$ $\max \left\{s_{1}, t_{1}\right\}, \sigma_{2}=\max \left\{s_{2}, t_{2}\right\}$; then

$$
\begin{aligned}
& \sigma_{1} \leq n_{1}+1, \\
& \sigma_{2} \leq n_{2}+1 .
\end{aligned}
$$

If (23) admits a pair of transcendental entire solutions of finite order, then

$$
\begin{aligned}
& \sigma_{1} \leq 1 \\
& \sigma_{2} \leq 1
\end{aligned}
$$

We give the following example to show that Theorem 13 is not valid for a pair of meromorphic solutions with infinite order of (23).

Example 14. Let $\left(f_{1}, f_{2}\right)=\left(e^{e^{z}}, e^{-e^{z}}\right)$; then $\left(f_{1}, f_{2}\right)$ are a pair of solutions of the system

$$
\begin{aligned}
& f_{1}^{\prime}(z)=e^{z} f_{2}(z+c)^{p}, \\
& f_{2}^{\prime}(z)=-e^{z} f_{1}(z+c)^{p},
\end{aligned}
$$

where $c$ is a complex constant and $p$ is a positive integer satisfying $e^{c}=-1 / p, p \geq 3$.

Theorem 15. Under the assumptions of Theorem 13, $\operatorname{let}\left(f_{1}, f_{2}\right)$ be a pair of transcendental meromorphic solutions of system

$$
\begin{aligned}
& \sum_{k_{1}=1}^{n_{1}} f_{1}^{\left(k_{1}\right)}(z) \\
& \quad=\frac{a_{0}(z)+a_{1}(z) f_{2} \circ p+\cdots+a_{s_{2}}(z)\left(f_{2} \circ p\right)^{s_{2}}}{b_{0}(z)+b_{1}(z) f_{2} \circ p+\cdots+b_{t_{2}}(z)\left(f_{2} \circ p\right)^{t_{2}}}, \\
& \sum_{k_{2}=1}^{n_{2}} f_{2}^{\left(k_{2}\right)}(z) \\
& \quad=\frac{e_{0}(z)+e_{1}(z) f_{1} \circ p+\cdots+e_{s_{1}}(z)\left(f_{1} \circ p\right)^{s_{1}}}{d_{0}(z)+d_{1}(z) f_{1} \circ p+\cdots+d_{t_{1}}(z)\left(f_{1} \circ p\right)^{t_{1}}},
\end{aligned}
$$

where $p(z)=q z^{k}+\cdots(q(\neq 0) \in \mathbb{C})$ of degree $k \geq 2$. Then

$$
\begin{aligned}
& T\left(r, f_{1}\right)=O\left((\log r)^{\varsigma+\varepsilon}\right), \\
& T\left(r, f_{2}\right)=O\left((\log r)^{\varsigma+\varepsilon}\right), \\
& k^{2} \sigma_{1} \sigma_{2} \leq\left(n_{1}+1\right)\left(n_{2}+1\right),
\end{aligned}
$$

where $\varepsilon>0$ and

$$
\varsigma=\frac{\log \left(n_{1}+1\right)\left(n_{2}+1\right)-\log \sigma_{1} \sigma_{2}}{2 \log k} .
$$

\section{Some Lemmas}

Lemma 16 (Valiron-Mohon'ko (see [24])). Let $f(z)$ be a meromorphic function. Then for all irreducible rational functions in $f$,

$$
R(z, f(z))=\frac{\sum_{i=0}^{m} a_{i}(z) f(z)^{i}}{\sum_{j=0}^{n} b_{j}(z) f(z)^{j}},
$$

with meromorphic coefficients $a_{i}(z), b_{j}(z)$, the characteristic function of $R(z, f(z))$ satisfies that

$$
T(r, R(z, f(z)))=d T(r, f)+O(\Psi(r)),
$$

where $d=\max \{m, n\}$ and $\Psi(r)=\max _{i, j}\left\{T\left(r, a_{i}\right), T\left(r, b_{j}\right)\right\}$.

Lemma 17 (see [14, p. 37]). If a meromorphic function $f$ satisfies

$$
\max \left\{\lambda(f), \lambda\left(\frac{1}{f}\right)\right\}<\rho(f),
$$

then $f$ is of regular growth.

Lemma 18 (see [15, p. 127]). The differential field

$$
\mathscr{L}_{f}=\{g \text { is meromorphic } \mid T(r, g)=S(r, f)\}
$$


is algebraically closed in the field of meromorphic functions in the complex plane. That is, any meromorphic function satisfying an algebraic equation over the field $\mathscr{L}_{f}$ actually belongs to $\mathscr{L}_{f}$.

Remark 19 (see [28, p. 249] and [16, p. 728 Remark]). The following observation

$$
N(r, f(q z))=N(|q| r, f)+O(1)
$$

holds for any meromorphic function $f$ and any nonzero complex constant $q$. Clearly, we can immediately obtain

$$
\lambda\left(\frac{1}{f(q z)}\right)=\lambda\left(\frac{1}{f}\right) .
$$

Similarly, we also have

$$
\begin{aligned}
N\left(r, \frac{1}{f(q z)}\right) & =N\left(|q| r, \frac{1}{f}\right)+O(1), \\
\lambda(f(q z)) & =\lambda(f) .
\end{aligned}
$$

Lemma 20 (see [29]). Let $f(z)$ be a transcendental meromorphic function and $p(z)=q z^{k}+\cdots$ a complex polynomial of degree $k>0$. For given $0<\delta<|q|$, let $\lambda=|q|+\delta, \mu=|q|-\delta$; then for given $\varepsilon>0$ and for $r$ large enough,

$$
(1-\varepsilon) T\left(\mu r^{k}, f\right) \leq T(r, f \circ p) \leq(1+\varepsilon) T\left(\lambda r^{k}, f\right) .
$$

Lemma 21. Suppose that $f_{1}, f_{2}$ are meromorphic functions of finite order $\rho\left(f_{1}\right), \rho\left(f_{2}\right), \rho\left(f_{1}\right), \rho\left(f_{2}\right)$ are positive integers satisfying (16), and let $q_{j_{t}} \in \mathbb{C} \backslash\{0\}, j_{t}=1, \ldots, n_{t}, t=1,2$.

(i) If $\rho_{1}=\rho\left(f_{1}\right)<\rho\left(f_{2}\right)=\rho_{2}$ and

$$
\sum_{j_{2}=1}^{n_{2}} q_{j_{2}}^{\rho_{2}} \neq 0
$$

then, $H(z)=\prod_{j_{1}=1}^{n_{1}} f_{1}\left(q_{j_{1}} z\right) \prod_{j_{2}=1}^{n_{2}} f_{2}\left(q_{j_{2}} z\right)$ satisfies $\rho(H)=\rho_{2}$.

(ii) If $\rho_{1}=\rho\left(f_{1}\right)>\rho\left(f_{2}\right)=\rho_{2}$ and

$$
\sum_{j_{1}=1}^{n_{1}} q_{j_{1}}^{\rho_{1}} \neq 0
$$

then, $H(z)=\prod_{j_{1}=1}^{n_{1}} f_{1}\left(q_{j_{1}} z\right) \prod_{j_{2}=1}^{n_{2}} f_{2}\left(q_{j_{2}} z\right)$ satisfies $\rho(H)=\rho_{1}$.

Remark 22. The following example shows that the conclusions are not valid if the condition $\rho_{1} \neq \rho_{2}$ is removed in Lemma 21.

Example 23. Let $\left(f_{1}, f_{2}\right)=\left(e^{z}, e^{-z}\right)$; then $\rho_{1}=\rho_{2}=1$ and $0=$ $\max \left\{\lambda\left(f_{t}\right), \lambda\left(1 / f_{t}\right)\right\}<1$. If $q_{j}, j=1,2, \ldots, n$, are complex constants and $n_{1}=n_{2}=n$ satisfy

$$
\sum_{j_{1}=1}^{n} q_{j_{1}}=\sum_{j_{1}=1}^{n} q_{j_{1}} \neq 0,
$$

then we have $H(z)=1$; that is, $\rho(H)=0 \neq 1$.
Proof. From the assumptions in Lemma 21 and by Hadamard Theorem, we have

$$
f_{t}(z)=z^{m_{t}} \frac{Q_{g}^{t}(z)}{Q_{h}^{t}(z)} e^{P_{t}(z)}, \quad t=1,2,
$$

where $m_{t} \in \mathbb{Z}, P_{1}(z), P_{1}(z)$ are polynomials of degree $\rho_{1}, \rho_{2}$, respectively, and $Q_{g}^{t}(z), Q_{h}^{t}(z)$ are the canonical products formed with nonzero zeros and poles of $f_{t}, t=1,2$, respectively. So, we have

$$
\begin{aligned}
H(z) & =\prod_{j_{1}=1}^{n_{1}}\left(q_{j_{1}} z\right)^{m_{1}} \frac{Q_{g}^{1}\left(q_{j_{1}} z\right)}{Q_{h}^{1}\left(q_{j_{1}} z\right)} e^{P_{1}\left(q_{j_{1}} z\right)} \prod_{j_{2}=1}^{n_{2}}\left(q_{j_{2}} z\right)^{m_{2}} \\
\cdot & \frac{Q_{g}^{2}\left(q_{j_{2}} z\right)}{Q_{h}^{2}\left(q_{j_{2}} z\right)} e^{P_{2}\left(q_{j_{2}} z\right)} .
\end{aligned}
$$

Denoting

$$
G_{j_{t}}(z)=\left(q_{j_{t}} z\right)^{m_{t}} \frac{Q_{g}^{t}\left(q_{j_{t}} z\right)}{Q_{h}^{t}\left(q_{j_{t}} z\right)}, \quad t=1,2,
$$

then we can rewrite $H(z)$ as the following form:

$$
\begin{aligned}
& H(z)=\exp \left\{\sum_{t=1}^{2}\left(\sum_{j_{t}=1}^{n_{t}} q_{j_{t}}^{\rho_{t}} a_{\rho_{t}} z^{\rho_{t}}+P_{\rho_{t}-1}(z)\right)\right\} \\
& \cdot \prod_{t=1}^{2} \prod_{j_{t}=1}^{n_{t}} G_{j_{t}}(z),
\end{aligned}
$$

where $a_{\rho_{t}}, t=1,2$, are the leading coefficient of $P_{t}(z)$ and $P_{\rho_{t}-1}(z)$ are polynomials of degree $\leq \rho_{t}-1$.

(i) $\rho_{1}<\rho_{2}$. By Remark 19, it follows from (16) that

$$
\begin{aligned}
\lambda(H) & \leq \max _{1 \leq j_{t} \leq n_{t}}\left\{\lambda\left(f_{t}\left(q_{j_{t}} z\right)\right), t=1,2\right\} \\
& =\max \left\{\lambda\left(f_{1}\right), \lambda\left(f_{2}\right)\right\}<\rho_{2}, \\
\lambda\left(\frac{1}{H}\right) & \leq \max _{1 \leq j_{t} \leq n_{t}}\left\{\lambda\left(\frac{1}{f_{t}\left(q_{j_{t}} z\right)}\right), t=1,2\right\} \\
& =\max \left\{\lambda\left(\frac{1}{f_{1}}\right), \lambda\left(\frac{1}{f_{2}}\right)\right\}<\rho_{2} .
\end{aligned}
$$

It follows from (44)-(46) that $\rho\left(G_{j_{t}}\right)<\rho_{2}$ for all $j_{t}=$ $1, \ldots, n_{t}, t=1,2$. Since $\sum_{j_{2}=1}^{n_{2}} q_{j_{2}}^{n_{2}} \neq 0$, we have $\rho(H)=\rho_{2}$.

(ii) $\rho_{1}>\rho_{2}$. By using the same argument as in (i), we can get $\rho(H)=\rho_{1}$ easily.

Thus, this completes the proof of Lemma 21.

Lemma 24 (see [2]). Let $f(z)$ be a transcendental meromorphic function of finite order and $c$ a nonzero complex constant. Then, we have

$$
T(r, f(z+c))=T(r, f(z))+S(r, f) .
$$


Lemma 25 (see [13, p. 37] or [12]). Let $f(z)$ be a nonconstant meromorphic function in the complex plane and $l$ a positive integer. Then

$$
\begin{aligned}
& N\left(r, f^{(l)}\right)=N(r, f)+l \bar{N}(r, f), \\
& T\left(r, f^{(l)}\right) \leq T(r, f)+l \bar{N}(r, f)+S(r, f) .
\end{aligned}
$$

Lemma 26 (see $[24,30])$. Let $g:(0,+\infty) \rightarrow R, h$ : $(0,+\infty) \rightarrow R$ be monotone increasing functions such that $g(r) \leq h(r)$ outside of an exceptional set $E$ with finite linear measure, or $g(r) \leq h(r), r \notin H \cup(0,1]$, where $H \subset(1, \infty)$ is a set of finite logarithmic measure. Then, for any $\alpha>1$, there exists $r_{0}$ such that $g(r) \leq h(\alpha r)$ for all $r \geq r_{0}$.

Lemma 27 (see [31]). Let $\psi(r)$ be a function of $r\left(r \geq r_{0}\right)$, positive, and bounded in every finite interval.

(i) Suppose that $\psi\left(\mu r^{m}\right) \leq A \psi(r)+B\left(r \geq r_{0}\right)$, where $\mu(\mu>0), m(m>1), A(A \geq 1)$, and $B$ are constants. Then $\psi(r)=O\left((\log r)^{\alpha}\right)$ with $\alpha=\log A / \log m$, unless $A=1$ and $B>0$; and if $A=1$ and $B>0$, then, for any $\varepsilon>0$, $\psi(r)=O\left((\log r)^{\varepsilon}\right)$.

(ii) Suppose that (with the notation of (i)) $\psi\left(\mu r^{m}\right) \geq$ $A \psi(r)\left(r \geq r_{0}\right)$. Then for all sufficiently large values of $r$, $\psi(r) \geq K(\log r)^{\alpha}$ with $\alpha=\log A / \log m$, for some positive constant $K$.

\section{Proofs of Theorem 8, Corollaries 9 and 10}

3.1. The Proof of Theorem 8. Let $G_{t}(z)=\prod_{j_{t}=1}^{n_{t}} f_{t}\left(q_{j_{t}} z\right), t=$ 1,2 . By applying Valiron-Mohon'ko theorem [24] to (15), we have

$$
\begin{aligned}
T\left(r, G_{1}\right)= & \max \left\{s_{2}, t_{2}\right\} T\left(r, f_{2}\right)+S\left(r, f_{1}\right) \\
& +S\left(r, f_{2}\right), \\
T\left(r, G_{2}\right)= & \max \left\{s_{1}, t_{1}\right\} T\left(r, f_{1}\right)+S\left(r, f_{1}\right) \\
& +S\left(r, f_{2}\right) .
\end{aligned}
$$

From (16), we can take constants $\xi_{t}, \delta_{t}$ such that

$$
\max \left\{\lambda\left(f_{t}\right), \lambda\left(\frac{1}{f_{t}}\right)\right\}<\xi_{t}<\delta_{t}<\rho\left(f_{t}\right), \quad t=1,2,
$$

and then we have

$$
\begin{aligned}
T\left(r, \frac{f_{t}^{\prime}}{f_{t}}\right) & =\bar{N}\left(r, f_{t}\right)+\bar{N}\left(r, \frac{1}{f_{t}}\right)+S\left(r, f_{t}\right) \\
& =O\left(r^{\xi_{t}}\right)+S\left(r, f_{t}\right), \quad t=1,2 .
\end{aligned}
$$

From the definitions of $G_{t}(t=1,2)$, similar to the above argument, we have

$$
\begin{aligned}
T\left(r, \frac{G_{t}^{\prime}}{G_{t}}\right)=N\left(r, \frac{G_{t}^{\prime}}{G_{t}}\right)+m\left(r, \frac{G_{t}^{\prime}}{G_{t}}\right) \\
\leq \sum_{j_{t}=1}^{n_{t}}\left(\bar{N}\left(r, f_{t}\left(q_{j_{t}} z\right)\right)+\bar{N}\left(r, \frac{1}{f_{t}\left(q_{j_{t}} z\right)}\right)\right) \\
\quad+S\left(r, f_{1}\right)+S\left(r, f_{2}\right) \\
=\sum_{j_{t}=1}^{n_{t}}\left(\bar{N}\left(\left|q_{j_{t}}\right| r, f_{t}(z)\right)+\bar{N}\left(\left|q_{j_{t}}\right| r, \frac{1}{f_{t}(z)}\right)\right) \\
\quad+S\left(r, f_{1}\right)+S\left(r, f_{2}\right) \\
=O\left(r^{\xi_{t}}\right)+S\left(r, f_{1}\right)+S\left(r, f_{2}\right) .
\end{aligned}
$$

From (16), we know that zeros and poles are Borel exceptions of $f_{t}(t=1,2)$, and from [32, Satz 13.4], we have that $f_{t}(t=$ $1,2)$ is of regular growth. Hence, there exists $r_{0}>0$ that $T\left(r, f_{t}\right)>r^{\delta_{t}}$ for $r>r_{0}$. So, we can get that

$$
\begin{aligned}
& T\left(r, \frac{G_{t}^{\prime}}{G_{t}}\right)=S\left(r, f_{1}\right)+S\left(r, f_{2}\right), \\
& T\left(r, \frac{f_{t}^{\prime}}{f_{t}}\right)=S\left(r, f_{1}\right)+S\left(r, f_{2}\right),
\end{aligned}
$$

$$
t=1,2 .
$$

Now, we rewrite system (15) as

$$
\begin{aligned}
& \frac{b_{t_{2}}(z)}{a_{s_{2}}(z)} G_{1}(z)=\frac{P_{2}\left(z, f_{2}\right)}{Q_{2}\left(z, f_{2}\right)}=u_{2}\left(z, f_{2}\right), \\
& \frac{d_{t_{1}}(z)}{e_{s_{1}}(z)} G_{2}(z)=\frac{P_{1}\left(z, f_{1}\right)}{Q_{1}\left(z, f_{1}\right)}=u_{1}\left(z, f_{1}\right),
\end{aligned}
$$

without loss of generality, assume that $P_{t}, Q_{t}$ are monic polynomials in $f_{t}$ with coefficients of growth $S\left(r, f_{1}\right), S\left(r, f_{2}\right)$, and set $F_{t}:=f_{t}^{\prime} / f_{t}, U_{t}:=u_{t}^{\prime} / u_{t}, t=1,2$; from (54), we have $T\left(r, U_{t}\right)=S\left(r, f_{1}\right)+S\left(r, f_{2}\right)$. And because

$$
\begin{aligned}
& \frac{P_{2}^{\prime} Q_{2}-P_{2} Q_{2}^{\prime}}{Q_{2}^{2}}=u_{2}^{\prime}=U_{2} u_{2}=\frac{U_{2} P_{2}}{Q_{2}}, \\
& \frac{P_{1}^{\prime} Q_{1}-P_{1} Q_{1}^{\prime}}{Q_{1}^{2}}=u_{1}^{\prime}=U_{1} u_{1}=\frac{U_{1} P_{1}}{Q_{1}},
\end{aligned}
$$

it follows that

$$
\begin{aligned}
& P_{2}^{\prime} Q_{2}-P_{2} Q_{2}^{\prime}=U_{2} P_{2} Q_{2}, \\
& P_{1}^{\prime} Q_{1}-P_{1} Q_{1}^{\prime}=U_{1} P_{1} Q_{1} .
\end{aligned}
$$

Substituting $f_{t}^{\prime}=F_{t} f_{t}, t=1,2$, to the above equalities and comparing the leading coefficients, we can get

$$
\begin{aligned}
& \left(s_{1}-t_{1}\right) F_{1}=U_{1}, \\
& \left(s_{2}-t_{2}\right) F_{2}=U_{2} .
\end{aligned}
$$


Solving the above system, we get

$$
\begin{aligned}
& u_{1}=\Gamma_{2} f_{1}(z)^{s_{1}-t_{1}}, \\
& u_{2}=\Gamma_{1} f_{2}(z)^{s_{2}-t_{2}},
\end{aligned}
$$

where $\Gamma_{t} \in \mathbb{C}, t=1,2$. From (54)-(58) and $b_{t_{2}}(z) \equiv$ $1, e_{t_{1}}(z) \equiv 1$, it follows that

$$
\begin{aligned}
& G_{1}(z)=\Gamma_{2} a_{s_{2}}(z) f_{2}(z)^{s_{2}-t_{2}}, \\
& G_{2}(z)=\Gamma_{1} e_{s_{1}}(z) f_{1}(z)^{s_{1}-t_{1}} .
\end{aligned}
$$

Substituting the above system into (15), we have

$$
\begin{aligned}
\Gamma_{2} a_{s_{2}}(z) f_{2}(z)^{s_{2}-t_{2}} & \\
= & \frac{a_{0}(z)+a_{1}(z) f_{2}(z)+\cdots+a_{s_{2}}(z) f_{2}(z)^{s_{2}}}{b_{0}(z)+b_{1}(z) f_{2}(z)+\cdots+f_{2}(z)^{t_{2}}}, \\
\Gamma_{1} e_{s_{1}}(z) f_{1}(z)^{s_{1}-t_{1}} & \\
= & \frac{e_{0}(z)+e_{1}(z) f_{1}(z)+\cdots+e_{s_{1}}(z) f_{1}(z)^{s_{1}}}{d_{0}(z)+d_{1}(z) f_{1}(z)+\cdots+f_{1}(z)^{t_{1}}},
\end{aligned}
$$

that is,

$$
\begin{aligned}
\Gamma_{2} a_{s_{2}} & (z) f_{2}(z)^{s_{2}}+\cdots+\Gamma_{2} a_{s_{2}}(z) b_{1} f_{2}(z)^{s_{2}-t_{2}+1} \\
& +\Gamma_{2} a_{s_{2}}(z) b_{0} f_{2}(z)^{s_{2}-t_{2}} \\
= & a_{0}(z)+a_{1}(z) f_{2}(z)+\cdots+a_{s_{2}}(z) f_{2}(z)^{s_{2}},
\end{aligned}
$$

where $s_{2} \geq t_{2}$, or

$$
\begin{aligned}
\Gamma_{2} a_{s_{2}} & (z) f_{2}(z)^{t_{2}}+\cdots+\Gamma_{2} a_{s_{2}}(z) b_{1} f_{2}(z) \\
& +\Gamma_{2} a_{s_{2}}(z) b_{0} \\
= & a_{0}(z) f_{2}(z)^{t_{2}-s_{2}}+a_{1}(z) f_{2}(z)^{t_{2}-s_{2}+1}+\cdots \\
& +a_{s_{2}}(z) f_{2}(z)^{t_{2}},
\end{aligned}
$$

where $t_{2}>s_{2}$. By regarding (61) or (62) as an algebraic equation in $f_{2}$ with coefficients of growth $S\left(r, f_{2}\right)$, then it follows by Lemma 18 that $\Gamma_{2}=1$. Moreover, if $s_{2} \neq 0$ and $t_{2} \neq 0$, we can get a contradiction with the condition that $R_{2}\left(z, f_{2}(z)\right)$ is irreducible in $f_{2}$. Thus, we have $s_{2}=0$ or $t_{2}=0$. Then it follows from (61) that $G_{1}(z)=a_{s_{2}}(z) f_{2}(z)^{s_{2}}$ or $G_{1}(z)=a_{0}(z) f_{2}(z)^{-t_{2}}$.

Similarly, we can get $G_{2}(z)=e_{s_{1}}(z) f_{1}(z)^{s_{1}}$ or $G_{2}(z)=$ $e_{0}(z) f_{1}(z)^{-t_{1}}$.

Thus, we complete the proof of Theorem 8 .

3.2. The Proof of Corollary 9. Let $\left(f_{1}, f_{2}\right)$ be a pair of transcendental solutions of (15) of finite order $\rho\left(f_{1}\right)=\rho_{1}, \rho\left(f_{2}\right)=\rho_{2}$.

(i) If $\rho_{1}<\rho_{2}$, we can exclude (17a) and (17b) easily by comparing the growth order of two sides of the equation

$$
\prod_{j_{1}=1}^{n_{1}} f_{1}\left(q_{j_{1}} z\right)=a_{s_{2}}(z) f_{2}(z)^{s_{2}} \text {. }
$$

Next, we will exclude (18a). Suppose that

$$
\prod_{j_{2}=1}^{n_{2}} f_{2}\left(q_{j_{2}} z\right)=e_{0}(z) f_{1}(z)^{s_{1}}
$$

From the assumptions of Lemma 21 and by Hadamard Theorem, we can write

$$
f_{t}(z)=z^{m_{t}} \frac{Q_{g}^{t}(z)}{Q_{h}^{t}(z)} e^{P_{t}(z)}, \quad t=1,2,
$$

where $m_{t} \in \mathbb{Z}, P_{1}(z), P_{2}(z)$ are polynomials of degree $\rho_{1}, \rho_{2}$, respectively, and $Q_{g}^{t}(z), Q_{h}^{t}(z)$ are the canonical products formed with nonzero zeros and poles of $f_{t}$, respectively. Substituting (65) into (64), we have

$$
\begin{aligned}
& \exp \left\{\left(\sum_{j_{2}=1}^{n_{2}} q_{j_{2}}^{\rho_{2}} a_{\rho_{2}} z^{\rho_{2}}+P_{\rho_{2}-1}(z)\right)\right\} \\
& \cdot \prod_{j_{2}=1}^{n_{2}}\left(q_{j_{2}} z\right)^{m_{2}} \frac{Q_{g}^{2}\left(q_{j_{2}} z\right)}{Q_{h}^{2}\left(q_{j_{2}} z\right)}=z^{s_{1} m_{1}} \frac{Q_{g}^{1}(z)^{s_{1}}}{Q_{h}^{1}(z)^{s_{1}}} e^{s_{1} P_{1}(z)} .
\end{aligned}
$$

Since $\sum_{j_{2}=1}^{n_{2}} q_{j_{2}}^{\rho_{2}} \neq 0$ and $\rho_{1}<\rho_{2}$, we can get a contradiction easily.

(ii) If $\rho_{1}>\rho_{2}$, we can exclude (17a) and (18a). By using the same argument as in (i) and combining the condition $\sum_{j_{1}=1}^{n_{1}} q_{j_{1}}^{\rho_{1}} \neq 0$ and $\max \left\{\rho\left(a_{s_{2}}\right), \rho\left(e_{s_{1}}\right)\right\}<\rho_{1}$, we can exclude (17b).

Therefore, we complete the proof of Corollary 9.

3.3. The Proof of Corollary 10. From the assumptions of Corollary 10 and (18b), we have

$$
\begin{aligned}
& f_{1}(z)^{t_{1}} f_{2}(z)^{t_{2}} \prod_{j_{2}=1}^{n_{2}} f_{2}\left(q_{j_{2}} z\right) \prod_{j_{1}=1}^{n_{1}} f_{1}\left(q_{j_{1}} z\right) \\
& =a_{0}(z) e_{0}(z) .
\end{aligned}
$$

We can rewrite (67) as the following form:

$$
\prod_{j_{2}=1}^{n_{2}+t_{2}} f_{2}\left(q_{j_{2}} z\right) \prod_{j_{1}=1}^{n_{1}+t_{1}} f_{1}\left(q_{j_{1}} z\right)=a_{0}(z) e_{0}(z)
$$

where $q_{n_{1}+1}=\cdots=q_{n_{1}+t_{1}}=q_{n_{2}+1}=\cdots=q_{n_{2}+t_{2}}=1$.

Suppose that $\rho_{1} \neq \rho_{2}$. Since

$$
\left(\sum_{j_{2}=1}^{n_{2}} q_{j_{2}}^{\rho_{2}}+t_{2}\right)\left(\sum_{j_{1}=1}^{n_{1}} q_{j_{1}}^{\rho_{1}}+t_{1}\right) \neq 0
$$

by using the similar method as in Lemma 21, from (68), we can get a contradiction with the assumption $\max \left\{\rho\left(a_{0}\right), \rho\left(e_{0}\right)\right\}<\min \left\{\rho_{1}, \rho_{2}\right\}$. Hence $\rho_{1}=\rho_{2}$.

Thus, we complete the proof of Corollary 10. 


\section{The Proof of Theorem 11}

Let $G_{t}(z)=\prod_{j_{t}=1}^{n_{t}} f_{t}\left(q_{j_{t}} z+c_{j_{t}}\right)$ and $g_{t}(z)=f_{t} \circ p, t=1,2$. By applying Valiron-Mohon'ko theorem [24] to (20), we have

$$
\begin{aligned}
T\left(r, G_{1}\right)= & \max \left\{s_{2}, t_{2}\right\} T\left(r, g_{2}\right)+S\left(r, g_{1}\right) \\
& +S\left(r, g_{2}\right), \\
T\left(r, G_{2}\right)= & \max \left\{s_{1}, t_{1}\right\} T\left(r, g_{1}\right)+S\left(r, g_{1}\right) \\
& +S\left(r, g_{2}\right) .
\end{aligned}
$$

From $(70)$ and the definitions of $G_{t}(t=1,2)$, similar to the above argument, we have

$$
\begin{aligned}
& T\left(r, \frac{G_{t}^{\prime}}{G_{t}}\right)=N\left(r, \frac{G_{t}^{\prime}}{G_{t}}\right)+m\left(r, \frac{G_{t}^{\prime}}{G_{t}}\right) \\
& \quad \leq \sum_{j_{t}=1}^{n_{t}}\left(\bar{N}\left(r, f_{t}\left(q_{j_{t}} z+c_{j_{t}}\right)\right)\right. \\
& \left.\quad+\bar{N}\left(r, \frac{1}{f_{t}\left(q_{j_{t}} z+c_{j_{t}}\right)}\right)\right)+S\left(r, g_{1}\right)+S\left(r, g_{2}\right) \\
& \quad \leq \sum_{j_{t}=1}^{n_{t}}\left(\bar{N}\left(\left|q_{j_{t}}\right| r+\left|c_{j_{t}}\right|, f_{t}\right)\right. \\
& \left.\quad+\bar{N}\left(\left|q_{j_{t}}\right| r+\left|c_{j_{t}}\right|, \frac{1}{f_{t}}\right)\right)+S\left(r, g_{1}\right)+S\left(r, g_{2}\right) .
\end{aligned}
$$

Since $T(r, f)$ is an increasing function for $r$ and $p(z)=q z^{k}+$ $\cdots$, hence, there exists $r_{0}>0$ such that for all $r>r_{0}>0$

$$
T\left(\left(|q|-\delta_{t}\right) r^{k}, f_{t}\right) \geq T\left(q_{t}^{*} r+c_{t}^{*}, f_{t}\right), \quad t=1,2,
$$

where $q_{t}^{*}=\max \left\{\left|q_{j_{t}}\right|, j_{t}=1,2, \ldots, n_{t}\right\}, c_{t}^{*}=\max \left\{\left|c_{j_{t}}\right|, j_{t}=\right.$ $\left.1,2, \ldots, n_{t}\right\}$, and $0<\delta_{t}<|q|, t=1,2$. Thus, it follows from the above inequality and Lemma 20 that

$$
\begin{gathered}
\frac{\bar{N}\left(q_{t}^{*} r+c_{t}^{*}, f_{t}\right)}{T\left(q_{t}^{*} r+c_{t}^{*}, f_{t}\right)}=\frac{\bar{N}\left(q_{t}^{*} r+c_{t}^{*}, f_{t}\right)}{T\left(r, g_{t}\right)} \frac{T\left(r, g_{t}\right)}{T\left(q_{t}^{*} r+c_{t}^{*}, f_{t}\right)} \\
\geq \frac{\bar{N}\left(q_{t}^{*} r+c_{t}^{*}, f_{t}\right)}{T\left(r, g_{t}\right)} \frac{T\left(\left(|q|-\delta_{t}\right) r^{k}, f_{t}\right)}{T\left(q_{t}^{*} r+c_{t}^{*}, f_{t}\right)}, \quad t=1,2 .
\end{gathered}
$$

From (16), we have $\bar{N}\left(q_{t}^{*} r+c_{t}^{*}, f_{t}\right) / T\left(q_{t}^{*} r+c_{t}^{*}, f_{t}\right) \rightarrow 0$ as $r \rightarrow \infty$ for $t=1,2$. Thus, we can get $\bar{N}\left(q_{t}^{*} r+\right.$ $\left.c_{t}^{*}, f_{t}\right) / T\left(r, g_{t}\right) \rightarrow 0$ as $r \rightarrow \infty$ for $t=1,2$; that is,

$$
\bar{N}\left(q_{t}^{*} r+c_{t}^{*}, f_{t}\right)=S\left(r, g_{t}\right), \quad t=1,2
$$

Similarly, we have

$$
\bar{N}\left(q_{t}^{*} r+c_{t}^{*}, \frac{1}{f_{t}}\right)=S\left(r, g_{t}\right), \quad t=1,2
$$

From (71)-(75), we have

$$
T\left(r, \frac{G_{t}^{\prime}}{G_{t}}\right)=S\left(r, g_{t}\right), \quad t=1,2 .
$$

Now, we rewrite system (20) as

$$
\begin{aligned}
& \frac{b_{t_{2}}(z)}{a_{s_{2}}(z)} G_{1}(z)=\frac{P_{2}\left(z, g_{2}\right)}{Q_{2}\left(z, g_{2}\right)}=u_{2}\left(z, g_{2}\right), \\
& \frac{d_{t_{1}}(z)}{e_{s_{1}}(z)} G_{2}(z)=\frac{P_{1}\left(z, g_{1}\right)}{Q_{1}\left(z, g_{1}\right)}=u_{1}\left(z, g_{1}\right),
\end{aligned}
$$

and, without loss of generality, assume that $P_{t}, Q_{t}$ are monic polynomials in $g_{t}$ with coefficients of growth $S\left(r, g_{1}\right), S\left(r, g_{2}\right)$. Thus, by using the same argument as in Theorem 8 , we can get the conclusion of Theorem 11 easily.

Therefore, this completes the proof of Theorem 11.

\section{Proofs of Theorems 13 and 15}

5.1. The Proof of Theorem 13. Let $\left(f_{1}, f_{2}\right)$ be a pair of transcendental meromorphic solutions of (23); by using Lemmas 16 and 25, it follows from (23) that

$$
\begin{aligned}
\sigma_{2} T\left(r, f_{2}(z+c)\right)= & T\left(r, \sum_{k_{1}=1}^{n_{1}} f_{1}^{\left(k_{1}\right)}(z)\right)+S\left(r, f_{1}\right) \\
& +S\left(r, f_{2}\right) \\
\leq & m\left(r, f_{1}\right)+\left(n_{1}+1\right) N\left(r, f_{1}\right) \\
& +S\left(r, f_{1}\right)+S\left(r, f_{2}\right) \\
\leq & \left(n_{1}+1\right) T\left(r, f_{1}\right)+S\left(r, f_{1}\right) \\
& +S\left(r, f_{2}\right), \\
\sigma_{1} T\left(r, f_{1}(z+c)\right)= & T\left(r, \sum_{k_{2}=1}^{n_{2}} f_{2}^{\left(k_{2}\right)}(z)\right)+S\left(r, f_{1}\right) \\
& +S\left(r, f_{2}\right) \\
\leq & m\left(r, f_{2}\right)+\left(n_{2}+1\right) N\left(r, f_{2}\right) \\
& +S\left(r, f_{1}\right)+S\left(r, f_{2}\right) \\
\leq & \left(n_{2}+1\right) T\left(r, f_{2}\right)+S\left(r, f_{1}\right) \\
& +S\left(r, f_{2}\right) .
\end{aligned}
$$

By Lemma 24, it follows that

$$
\begin{aligned}
& \sigma_{2} T\left(r, f_{2}\right) \leq\left(n_{1}+1\right) T\left(r, f_{1}\right)+S\left(r, f_{1}\right)+S\left(r, f_{2}\right) \\
& \sigma_{1} T\left(r, f_{1}\right) \leq\left(n_{2}+1\right) T\left(r, f_{2}\right)+S\left(r, f_{1}\right)+S\left(r, f_{2}\right) .
\end{aligned}
$$

From the above inequalities and the fact that $f_{1}, f_{2}$ are transcendental functions, then we have $\sigma_{1} \leq n_{1}+1$ and $\sigma_{2} \leq n_{2}+1$. If $f_{1}, f_{2}$ are entire functions, we can get $\sigma_{1} \leq 1$ and $\sigma_{2} \leq 1$, easily.

Hence, this completes the proof of Theorem 13. 
5.2. The Proof of Theorem 15. By using the same argument as in Theorem 13, we can get

$$
\begin{aligned}
\sigma_{2} T\left(r, f_{2} \circ p\right) \leq & \left(n_{1}+1\right) T\left(r, f_{1}\right)+S\left(r, f_{1}\right) \\
& +S\left(r, f_{2}\right), \\
\sigma_{1} T\left(r, f_{1} \circ p\right) \leq & \left(n_{2}+1\right) T\left(r, f_{2}\right)+S\left(r, f_{1}\right) \\
& +S\left(r, f_{2}\right) .
\end{aligned}
$$

Since $p(z)=q z^{k}+\cdots$, by Lemma 20, we can get that, for $\vartheta_{t}=|q|-\delta_{t}(>0), t=1,2$ and sufficiently large $r$,

$$
\begin{array}{r}
\sigma_{1}(1-\varepsilon) T\left(\vartheta_{1} r^{k}, f_{1}\right) \leq\left(n_{1}+1\right)(1+o(1)) T\left(r, f_{2}\right), \\
r \notin E_{1}, \\
\sigma_{2}(1-\varepsilon) T\left(\vartheta_{2} r^{k}, f_{2}\right) \leq\left(n_{2}+1\right)(1+o(1)) T\left(r, f_{1}\right), \\
r \notin E_{2},
\end{array}
$$

where $E_{1}, E_{2}$ are two sets of finite linear measure. By Lemma 26, we have that, for any given $\gamma_{1}>1, \gamma_{2}>1$ and for sufficiently large $r$,

$$
\begin{aligned}
& \sigma_{1}(1-\varepsilon) T\left(\vartheta_{1} r^{k}, f_{1}\right) \\
& \quad \leq\left(n_{1}+1\right)(1+o(1)) T\left(\gamma_{1} r, f_{2}\right), \\
& \sigma_{2}(1-\varepsilon) T\left(\vartheta_{2} r^{k}, f_{2}\right) \\
& \quad \leq\left(n_{2}+1\right)(1+o(1)) T\left(\gamma_{2} r, f_{1}\right) ;
\end{aligned}
$$

that is,

$$
\begin{aligned}
& T\left(\frac{\vartheta_{1}}{\gamma_{1}^{k}} R_{1}^{k}, f_{1}\right) \leq \frac{\left(n_{1}+1\right)(1+o(1))}{\sigma_{1}(1-\varepsilon)} T\left(R_{1}, f_{2}\right), \\
& T\left(\frac{\vartheta_{2}}{\gamma_{2}^{k}} R_{2}^{k}, f_{2}\right) \leq \frac{\left(n_{2}+1\right)(1+o(1))}{\sigma_{2}(1-\varepsilon)} T\left(R_{2}, f_{1}\right),
\end{aligned}
$$

where $R_{1}=\gamma_{1} r$ and $R_{2}=\gamma_{2} r$. From (83) and (84), we have

$$
\begin{aligned}
& T\left(\frac{\vartheta_{1} \vartheta_{2}^{k}}{\left.\gamma_{1}^{k} \gamma_{2}^{2 k} r^{2 k}, f_{1}\right)}\right. \\
& \quad \leq \frac{\left(n_{1}+1\right)(1+o(1))}{\sigma_{1}(1-\varepsilon)} \frac{\left(n_{2}+1\right)(1+o(1))}{\sigma_{2}(1-\varepsilon)} T\left(r, f_{1}\right), \\
& T\left(\frac{\vartheta_{2} \vartheta_{1}^{k}}{\left.\gamma_{2}^{k} \gamma_{1}^{2 k} r^{2 k}, f_{2}\right)}\right. \\
& \quad \leq \frac{\left(n_{2}+1\right)(1+o(1))}{\sigma_{2}(1-\varepsilon)} \frac{\left(n_{1}+1\right)(1+o(1))}{\sigma_{1}(1-\varepsilon)} T\left(r, f_{2}\right) .
\end{aligned}
$$

And since $k \geq 2$, we get that $\sigma_{1} \sigma_{2} \leq\left(n_{1}+1\right)\left(n_{2}+1\right)$. Then it follows from (85) and by Lemma 27 that

$$
\begin{aligned}
& T\left(r, f_{1}\right)=O\left((\log r)^{\varsigma_{1}}\right), \\
& T\left(r, f_{2}\right)=O\left((\log r)^{\varsigma_{1}}\right),
\end{aligned}
$$

where

$$
\varsigma_{1}=\frac{\log \left(n_{1}+1\right)\left(n_{2}+1\right)-\log \sigma_{1} \sigma_{2}+2 \log (1+o(1))-2 \log (1-\varepsilon)}{2 \log k}=\frac{\log \left(n_{1}+1\right)\left(n_{2}+1\right)-\log \sigma_{1} \sigma_{2}}{2 \log k}+\varepsilon_{1} .
$$

Set $\varsigma=\left(\log \left(n_{1}+1\right)\left(n_{2}+1\right)-\log \sigma_{1} \sigma_{2}\right) / 2 \log k$; then we have

$$
\begin{aligned}
& T\left(r, f_{1}\right)=O\left((\log r)^{\varsigma+\varepsilon_{1}}\right), \\
& T\left(r, f_{2}\right)=O\left((\log r)^{\varsigma+\varepsilon_{1}}\right) .
\end{aligned}
$$

Next, we will prove that $k^{2} \sigma_{1} \sigma_{2} \leq\left(n_{1}+1\right)\left(n_{2}+1\right)$. Suppose that $k^{2} \sigma_{1} \sigma_{2}>\left(n_{1}+1\right)\left(n_{2}+1\right)$; then we can get that $\varsigma=\left(\log \left(n_{1}+\right.\right.$ $\left.1)\left(n_{2}+1\right)-\log \sigma_{1} \sigma_{2}\right) / 2 \log k<1$. For sufficiently small $\varepsilon_{1}>0$, we have $\varsigma_{1}=\varsigma+\varepsilon_{1}<1$. This contradicts the condition on the transcendence of $f_{1}, f_{2}$.

Thus, the proof of Theorem 15 is completed.

\section{Conflict of Interests}

The authors declare that there is no conflict of interests regarding the publication of this paper.

\section{Acknowledgments}

The authors were supported by the NSF of China (11171170, 11301233, and 11561033), the Natural Science Foundation of Jiangxi Province in China (20132BAB211001, 20151BAB201008), and the Foundation of Education Department of Jiangxi (GJJ14644) of China.

\section{References}

[1] D. C. Barnett, R. G. Halburd, W. Morgan, and R. J. Korhonen, "Nevanlinna theory for the $q$-difference operator and meromorphic solutions of $q$-difference equations," Proceedings of the Royal Society of Edinburgh Section A: Mathematics, vol. 137, no. 3, pp. 457-474, 2007.

[2] Y.-M. Chiang and S.-J. Feng, "On the Nevanlinna characteristic of $f(z+\eta)$ and difference equations in the complex plane," The Ramanujan Journal, vol. 16, no. 1, pp. 105-129, 2008.

[3] R. G. Halburd and R. J. Korhonen, "Difference analogue of the lemma on the logarithmic derivative with applications to 
difference equations," Journal of Mathematical Analysis and Applications, vol. 314, no. 2, pp. 477-487, 2006.

[4] Z. X. Chen, Z. B. Huang, and X. M. Zheng, "On properties of difference polynomials," Acta Mathematica Scientia B, vol. 31, no. 2, pp. 627-633, 2011.

[5] R. G. Halburd and R. J. Korhonen, "Nevanlinna theory for the difference operator," Annales Academiae Scientiarum Fennicae. Mathematica, vol. 31, no. 2, pp. 463-478, 2006.

[6] J. Heittokangas, R. Korhonen, I. Laine, J. Rieppo, and J. Zhang, "Value sharing results for shifts of meromorphic functions, and sufficient conditions for periodicity," Journal of Mathematical Analysis and Applications, vol. 355, no. 1, pp. 352-363, 2009.

[7] I. Laine and C.-C. Yang, "Value distribution of difference polynomials," Proceedings of the Japan Academy-Series A, Mathematical Sciences, vol. 83, no. 8, pp. 148-151, 2007.

[8] K. Liu and L.-Z. Yang, "Value distribution of the difference operator," Archiv der Mathematik, vol. 92, no. 3, pp. 270-278, 2009.

[9] J. Zhang and R. Korhonen, "On the Nevanlinna characteristic of $f(\mathrm{qz})$ and its applications," Journal of Mathematical Analysis and Applications, vol. 369, no. 2, pp. 537-544, 2010.

[10] X.-M. Zheng and Z.-X. Chen, "Some properties of meromorphic solutions of $q$-difference equations," Journal of Mathematical Analysis and Applications, vol. 361, no. 2, pp. 472-480, 2010.

[11] W. K. Hayman, Meromorphic Functions, Clarendon Press, Oxford, UK, 1964.

[12] L. Yang, Value Distribution Theory, Springer, Berlin, Germany, 1993.

[13] H. X. Yi and C. C. Yang, Uniqueness Theory of Meromorphic Functions, Kluwer Academic Publishers, Dordrecht, The Netherlands, 2003, Chinese Original: Science Press, Beijing, China, 1995.

[14] J. Heittokangas, R. Korhonen, I. Laine, J. Rieppo, and K. Tohge, "Complex difference equations of Malmquist type," Computational Methods and Function Theory, vol. 1, no. 1, pp. 27-39, 2001.

[15] G. G. Gundersen, J. Heittokangas, I. Laine, J. Rieppo, and D. Yang, "Meromorphic solutions of generalized Schröder equations," Aequationes Mathematicae, vol. 63, no. 1-2, pp. 110$135,2002$.

[16] X. Zheng and Z. Chen, "On properties of $q$-difference equations," Acta Mathematica Scientia Series B, vol. 32, no. 2, pp. 724734, 2012.

[17] L. Y. Gao, "On meromorphic solutions of a type of system of composite functional equations," Acta Mathematica Scientia, vol. 32, no. 2, pp. 800-806, 2012.

[18] L. Y. Gao, "Systems of complex difference equations of Malmquist type," Acta Mathematica Sinica. Chinese Series, vol. 55, no. 2, pp. 293-300, 2012.

[19] L. Y. Gao, "Estimates of $N$-function and $m$-function of meromorphic solutions of systems of complex difference equations," Acta Mathematica Scientia B, vol. 32, no. 4, pp. 1495-1502, 2012.

[20] H.-Y. Xu, B.-X. Liu, and K.-Z. Tang, "Some properties of meromorphic solutions of systems of complex $q$-shift difference equations," Abstract and Applied Analysis, vol. 2013, Article ID 680956, 6 pages, 2013.

[21] H.-Y.Xu and Z.-X. Xuan, "Some properties of solutions of a class of systems of complex $q$-shift difference equations," Advances in Difference Equations, vol. 2013, article 271, 2013.

[22] S. A. Gao, Z. X. Chen, and T. W. Chen, Complex Oscillation Theory of Linear Differential Equations, Huazhong University of Science and Technology Press, Wuhan, China, 1997.
[23] J. He and X. M. Zheng, "The iterated order of meromorphic solutions of some classes of higher order linear differential equations," Journal of Jiangxi Normal University. Natural Sciences, vol. 36, no. 6, pp. 584-588, 2012.

[24] I. Laine, Nevanlinna Theory and Complex Differential Equations, Walter de Gruyter, Berlin, Germany, 1993.

[25] J. Tu, H. X. Huang, H. Y. Xu, and C. F. Chen, "The order and type of meromorphic functions and analytic functions in the unit disc," Journal of Jiangxi Normal University. Natural Science, vol. 37, no. 5, pp. 449-452, 2013.

[26] L. W. Liao, "The new developments in the research of nonlinear complex differential equations," Journal of Jiangxi Normal University. Natural Sciences, vol. 39, no. 4, pp. 331-339, 2015.

[27] K. Liu and X. J. Dong, "Some results related to complex differential-difference equations of certain types," Bulletin of the Korean Mathematical Society, vol. 51, no. 5, pp. 1453-1467, 2014.

[28] W. Bergweiler, K. Ishizaki, and N. Yanagihara, "Meromorphic solutions of some functional equations," Methods and Applications of Analysis, vol. 5, no. 3, pp. 248-259, 1998, Correction: Methods and Applications of Analysis, vol. 6, no. 4, pp. 617-618, 1999.

[29] R. Goldstein, "Some results on factorisation of meromorphic functions," Journal of the London Mathematical Society, vol. 4, no. 2, pp. 357-364, 1971.

[30] G. G. Gundersen, "Finite order solutions of second order linear differential equations," Transactions of the American Mathematical Society, vol. 305, no. 1, pp. 415-429, 1988.

[31] R. Goldstein, "On meromorphic solutions of certain functional equations," Aequationes Mathematicae, vol. 18, no. 1-2, pp. 112157, 1978.

[32] G. Jank and L. Volkmann, Einführung in die Theorie der Ganzen und Meromorphen Funktionen mit Anwendungen auf Differentialgleichungen, Birkhäuser, Basel, Switzerland, 1985. 


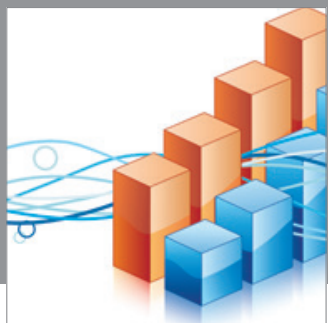

Advances in

Operations Research

mansans

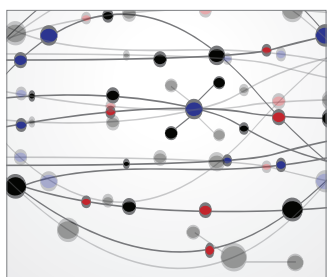

The Scientific World Journal
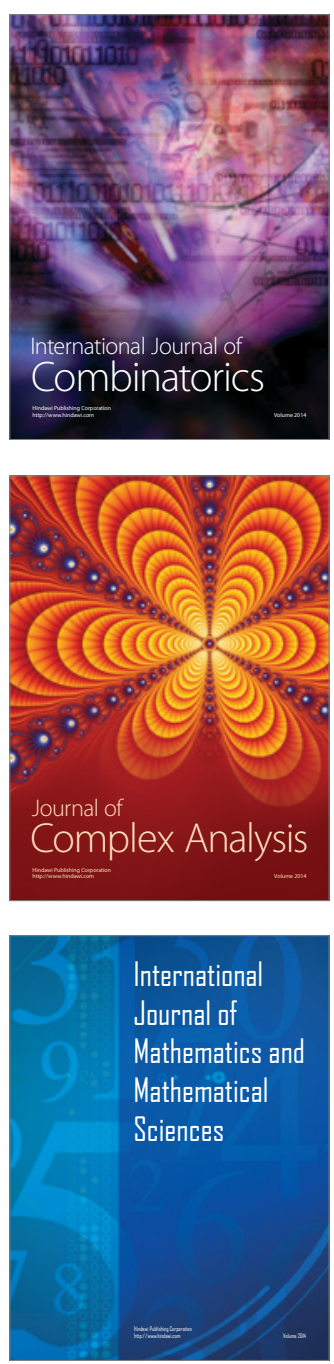
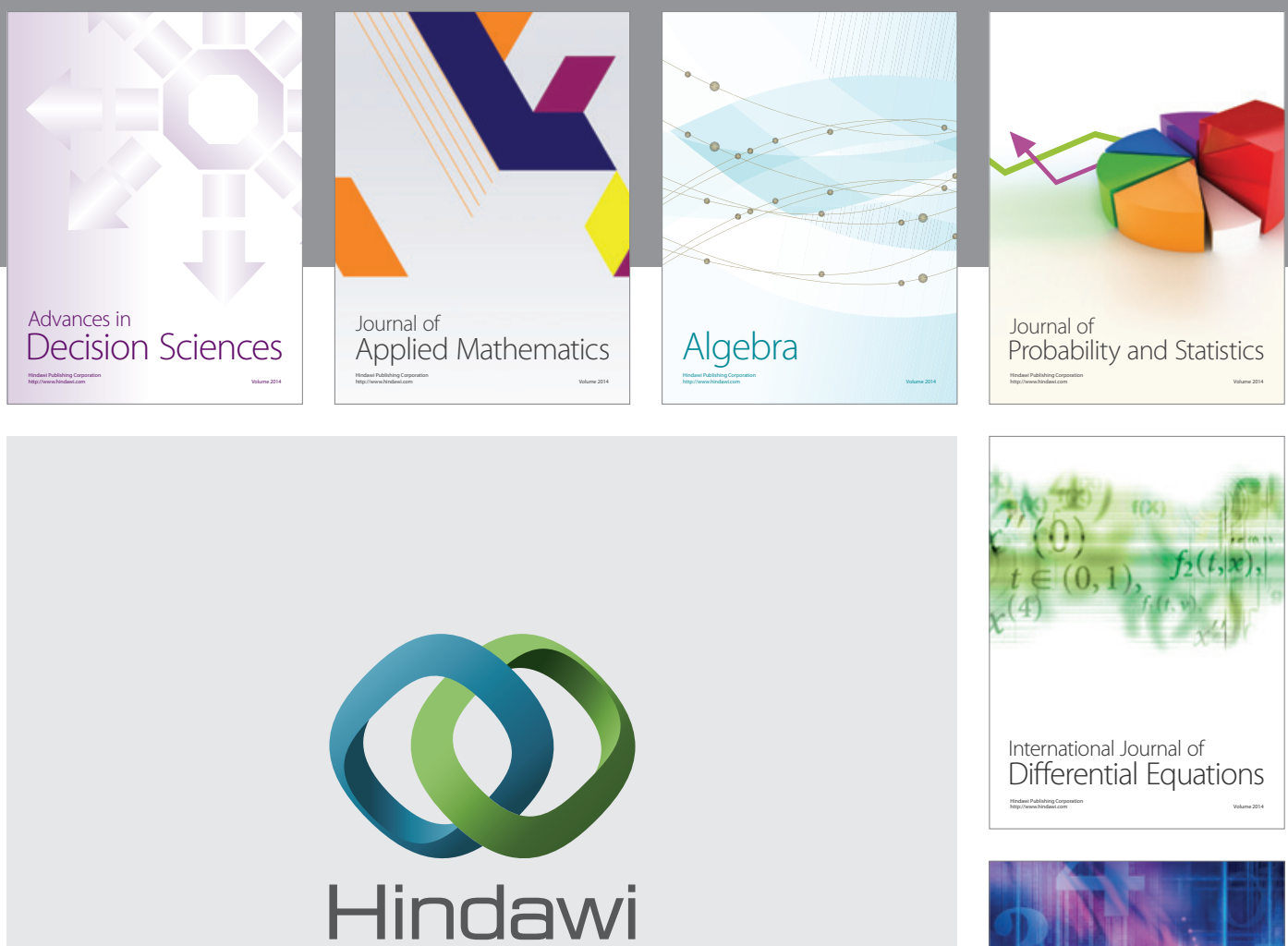

Submit your manuscripts at http://www.hindawi.com
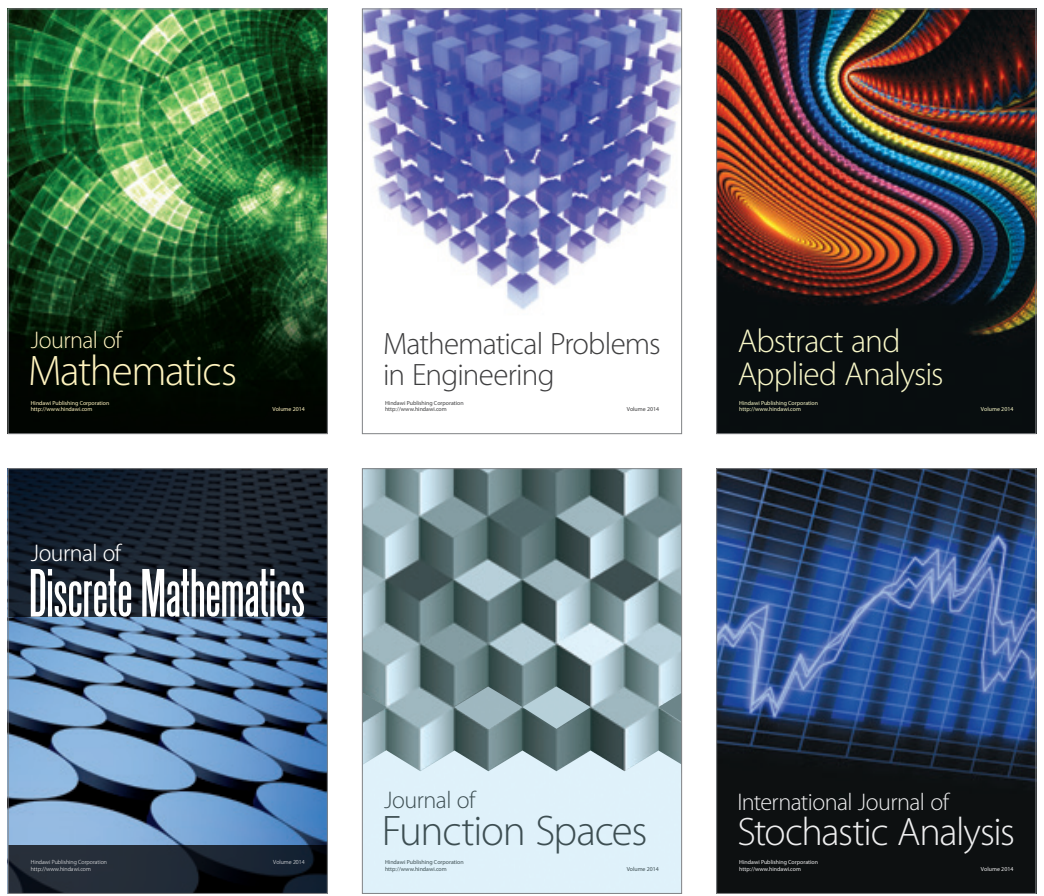

Journal of

Function Spaces

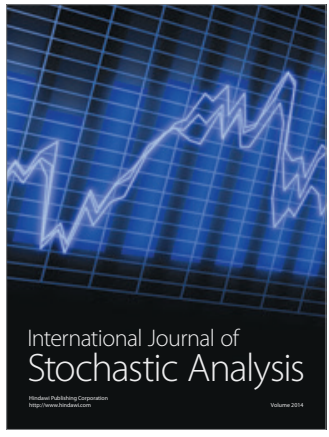

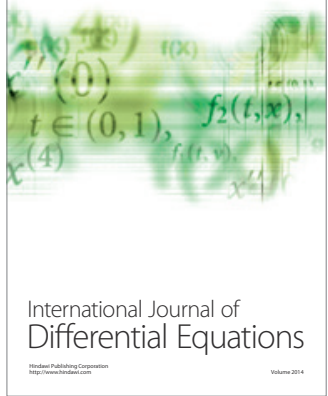
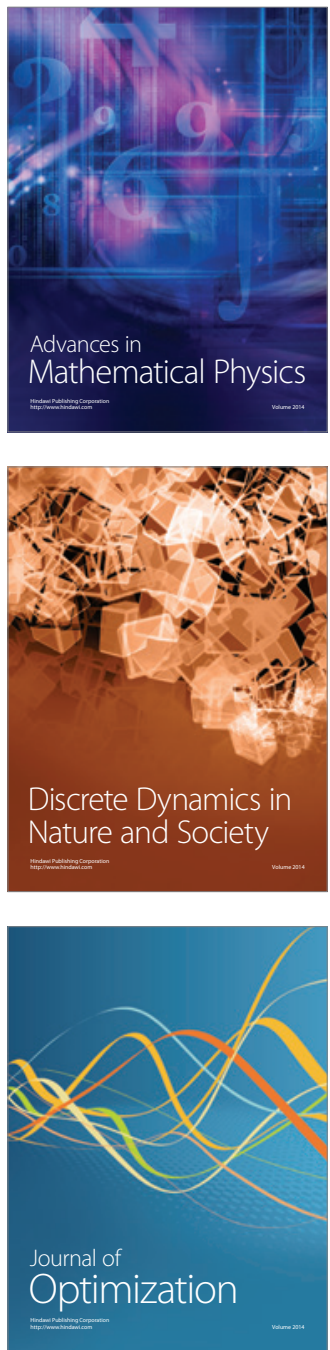